\title{
Clonidine for sedation in the critically ill: a systematic review and meta-analysis (protocol)

Gennie Jing Wang ${ }^{1}$, Emilie Belley-Coté ${ }^{2}$, Lisa Burry ${ }^{3,4}$, Mark Duffett ${ }^{2,5}$, Timothy Karachi ${ }^{1,6}$, Dan Perri ${ }^{1,7}$,

Waleed Alhazzani ${ }^{1,2}$, Frederick D'Aragon ${ }^{2}$, Hannah Wunsch ${ }^{8,9,10}$ and Bram Rochwerg ${ }^{1,2^{*}}$

\begin{abstract}
Background: Management and choice of sedation is important during critical illness in order to reduce patient suffering and to facilitate the delivery of care. Unfortunately, medications traditionally used for sedation in the intensive care unit (ICU) such as benzodiazepines and propofol are associated with significant unwanted effects. Clonidine is an alpha-2 selective adrenergic agonist that may have a role in optimizing current sedation practices in the pediatric and adult critically ill populations by potentially minimizing exposure to other sedative agents.

Methods/design: We will search MEDLINE, EMBASE, CINAHL, ACPJC, the Cochrane trial registry, World Health Organization International Clinical Trials Registry Platform (WHO ICTRP), and clinicaltrials.gov for eligible observational studies and randomized controlled trials investigating the use of clonidine as an adjunctive or stand-alone sedative agent in patients requiring invasive mechanical ventilation. Our primary outcome is the duration of mechanical ventilation. Secondary outcomes include the following, listed by priority: duration of sedation infusions, dose of sedation used, level of sedation, incidence of withdrawal from other sedatives, delirium incidence, ICU and hospital length of stay, use and duration of non-invasive ventilation, and all-cause ICU and hospital mortality. We will also capture unwanted effects potentially associated with clonidine administration such as clinically significant hypotension or bradycardia, clonidine withdrawal, self-extubation, and the accidental removal of central intravenous lines and arterial lines.

We will not apply any publication date, language, or journal restrictions. Two reviewers will independently screen and identify eligible studies using predefined eligibility criteria and then review full reports of all potentially relevant citations. A third reviewer will resolve disagreements if consensus cannot be achieved. We will use Review Manager (RevMan) to pool effect estimates from included studies across outcomes. We will present the results as relative risk (RR) with $95 \%$ confidence intervals (Cl) for dichotomous outcomes and as mean difference (MD) or standardized mean difference (SMD) for continuous outcomes with $95 \% \mathrm{Cl}$. We will assess the quality of evidence using the Grading of Recommendations, Assessment, Development and Evaluation (GRADE) approach.
\end{abstract}

Discussion: The aim of this systematic review is to summarize the evidence on the efficacy and safety of clonidine as a sedative agent in the critically ill population.

Systematic review registration: PROSPERO CRD42015019365.

Keywords: Clonidine, Systematic review, Sedation, Delirium, Mechanical ventilation, Weaning

\footnotetext{
* Correspondence: rochwerg@mcmaster.ca

'Department of Medicine, Faculty of Health Sciences, McMaster University,

Hamilton, Ontario, Canada

${ }^{2}$ Department of Clinical Epidemiology and Biostatistics, McMaster University,

Hamilton, Ontario, Canada

Full list of author information is available at the end of the article
}

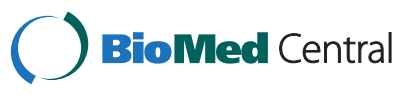

(c) 2015 Jing Wang et al. Open Access This article is distributed under the terms of the Creative Commons Attribution 4.0 International License (http://creativecommons.org/licenses/by/4.0/), which permits unrestricted use, distribution, and reproduction in any medium, provided you give appropriate credit to the original author(s) and the source, provide a link to the Creative Commons license, and indicate if changes were made. The Creative Commons Public Domain Dedication waiver (http://creativecommons.org/publicdomain/zero/1.0/) applies to the data made available in this article, unless otherwise stated. 


\section{Background}

\section{Description of the condition}

The use of sedative agents in the mechanically ventilated, critically ill population is well established, and the rationale for their use is multifaceted. Sedation is important in reducing patient discomfort and suffering through alleviation of pain and anxiety [1]. Sedation also reduces the autonomic hyperactivity that frequently results when these factors are poorly controlled [2]. Achieving appropriate levels of sedation also facilitates the delivery of care and enhances patient safety by reducing the risks of accidental removal of life-sustaining interventions such as endotracheal tubes or central venous catheters [3].

Despite the benefits of sedation, oversedation is unfavorable, as it minimizes patient interaction with caregivers and family members, delays weaning from the ventilator, may influence risk of delirium, and unnecessarily prolongs intensive care unit (ICU) stay [4]. The optimal sedation regimen should therefore provide adequate sedation with a rapid onset, allow timely recovery after cessation with minimal drug accumulation, lead to minimal adverse effects, and be relatively inexpensive. As a result, guidelines recommend the minimization of intravenous sedation infusions and the use of as small doses of sedatives as possible [5].

Commonly used and widely available ICU sedative agents include propofol and benzodiazepines (such as lorazepam, midazolam, and diazepam) [5-7]. These sedative agents are frequently used in conjunction with analgesic medications, such as opioids [8].

Importantly, the use of these sedative agents is not without adverse effects. Propofol is known to cause hypotension in a significant number of patients [9]. Propofol infusion syndrome, although a rare complication associated with higher doses and a longer duration of treatment, manifests with arrhythmias, rhabdomyolysis, acute kidney injury, myocardial dysfunction and is associated with a very high mortality rate [10]. Benzodiazepines may cause respiratory and cardiovascular depression as well as unintended excessive sedation secondary to drug accumulation in adipose tissue [11]. Benzodiazepines have also been shown to be associated with increased rates of ICU delirium and prolonged mechanical ventilation compared to other nonbenzodiazepine sedative agents [12].

Dexmedetomidine is a centrally acting alpha- 2 agonist that has sedative properties. Although smaller trials have shown that it may reduce the duration of mechanical ventilation and delirium with a decrease need for alternative sedatives [13-15], it is not universally available mostly related to cost.

\section{Description of intervention}

Clonidine is a centrally acting alpha-2 selective adrenergic agonist similar in action to dexmedetomidine.
Traditionally, clonidine has been used to treat attention deficit hyperactivity disorder (ADHD) [16], opioid and alcohol withdrawal [17, 18], hypertension, vasomotor menopausal symptoms, and for neuraxial anesthesia via epidural administration $[19,20]$. In the critically ill pediatric population, clonidine is frequently used as a sedative agent, particularly as an adjunctive agent when there is an inadequate response to opioids and benzodiazepines, or to help facilitate weaning from mechanical ventilation [21]. The evidence to support the use of clonidine in the critically ill adult population is less clear. Overall, the current clinical use of clonidine in the critically ill is quite variable [4].

Data supporting the use of clonidine as a sedative agent in the ICU setting remains limited. Known side effects of clonidine include hypotension and rarely bradycardia, as well as rebound tachycardia and hypertension after clonidine withdrawal. Clonidine can be administered via oral, transdermal, or intravenous route. However, only the oral and transdermal formulations are available in North America. Generic versions of clonidine are available, making this intervention extremely inexpensive.

\section{How the intervention might work}

Clonidine is a centrally acting alpha-2 selective adrenergic agonist. It has been postulated that clonidine exerts its sedative effects via stimulation of the pre-synaptic alpha- 2 adrenoceptors of the locus coeruleus, decreasing norepinephrine release [22]. Clonidine also has action on the cholinergic, purinergic, and serotonergic pathways, resulting in analgesia [22].

\section{Why it is important to do this review}

The current literature on sedation practices in the critically ill patient population lacks comprehensive summary data on the efficacy of clonidine as a sedative agent. One systematic review, which focused only on pediatric ICU patients, found that adjunctive clonidine use decreased the requirement for other sedative agents, decreased withdrawal symptoms when weaning off benzodiazepines and/or opiates, and was associated with minimal clinically significant adverse effects [21]. Another systematic review specifically examined the role of alpha-2 agonists on sedation in the mechanically ventilated patient population, however focused only on the role of dexmedetomidine [23].

Clonidine is an attractive alternative to other sedating medications given its ease of administration and its improved safety profile. Despite the potential concern regarding hypotension in comparison to dexmedetomidine, clonidine is associated with significant advantages including the availability of an oral formulation and significant cost savings. 


\section{Objectives}

We plan to conduct a systematic review of all observational studies, quasi-experimental studies, and RCTs that investigated the use of clonidine as an adjunctive or stand-alone sedative agent in the critically ill population.

\section{Methods/design}

Types of studies

We plan to include all observational cohort studies that included a control or comparator arm, quasi-experimental studies, and RCTs reporting the use of clonidine as a sedative agent in mechanically ventilated patients. We will exclude case reports, case series, or observational studies that did not include a control/comparator. We will impose no methodological quality restrictions.

\section{Types of participants}

The population of interest includes all patients, including children (under 18 years of age) and adults (18 years of age or older) who require invasive mechanical ventilation (IMV) and sedation. We will exclude studies enrolling exclusively neonates or those that take place exclusively in the neonatal intensive care unit (NICU). If a study includes both IMV and non-invasive ventilation (NIV) patients, we will include the study, but exclude all NIV patients if possible.

\section{Types of interventions}

The intervention of interest is the use of clonidine as a sedative agent for more than $24 \mathrm{~h}$. We will include any mode of delivery of clonidine other than intrathecal. We require the studies to have used clonidine either as an adjunctive or as a stand-alone sedative agent. The comparators or control group will be any standard sedation regimen that does not include clonidine, such as propofol, benzodiazepines, dexmedetomidine, and/or opioids. We will exclude studies using clonidine as a pre-medication for anesthesia or studies in which patients were given clonidine for an alternate indication.

\section{Types of outcome measures}

Our primary outcome is the duration of mechanical ventilation. Secondary outcomes include the following, listed by priority: duration of sedation infusions, dose of sedation used, level of sedation (as assessed by standardized sedation scores such as the Richmond Agitation-Sedation Scale or COMFORT score), incidence of withdrawal from other sedatives, delirium incidence (using the Confusion Assessment Method for the Intensive Care Unit (CAMICU), ICU and hospital length of stay, use and duration of NIV, and all-cause ICU and hospital mortality. We will also capture unwanted effects potentially associated with clonidine administration such as clinically significant hypotension or bradycardia (with end-organ dysfunction or requiring intervention), clonidine withdrawal (rebound tachycardia or hypertension), self-extubation, and the accidental removal of central intravenous lines and arterial lines. We will also capture unwanted effects such as clinically significant hypotension or bradycardia (with endorgan dysfunction or requiring intervention), clonidine withdrawal (rebound tachycardia or hypertension), selfextubation, and the accidental removal of central intravenous lines and arterial lines.

\section{Search methods for identification of studies}

We will search the following electronic databases: MEDLINE, EMBASE, ACPJC, CINAHL, and the Cochrane trial registry for eligible articles with no date or language restriction. See appendix for MEDLINE search strategy [Additional file 1]. Keyword search terms include clonidine, sedation, critically ill, mechanical ventilation, delirium, and withdrawal.

\section{Searching other resources}

Two reviewers will independently hand-search the references of review articles and systematic reviews on the same topic for eligible articles. In addition, we will search for unpublished or ongoing trials on the WHO International Clinical Trials Registry (WHO ICTRP), current controlled trials metaregister of controlled trials, clinicaltrials.gov database, and conference proceedings citation index within the last 2 years for the Society of Critical Care Medicine (SCCM), Canadian Critical Care Society, the European Society of Intensive Care Medicine (ESICM), the International Society of Intensive Care and Emergency Medicine (ISICEM), American Thoracic Society (ATS), and the World Federation of Pediatric Intensive and Critical Care Societies.

\section{Data collection and analysis}

After identification of potentially relevant articles, three reviewers (JW, EBC, BR) working in pairs will independently screen all citations and references using specific eligibility criteria. Disagreements will be resolved by discussion and consensus with the help of the third reviewer if needed.

\section{Data extraction and management}

Data extraction will be done independently and in duplicate using pre-designed data abstraction forms (see "Observational abstraction [Additional file 2]" and "RCT abstraction" [Additional file 3]). Abstracted data will include the study title, first author, relevant demographic data, intervention and control, results for outcomes of interest, and information on the methodological quality for each study. A third reviewer will resolve inconsistent data extraction between the two reviewers. 


\section{Assessment of risk of bias in included studies}

Two reviewers will independently assess the risk of bias for each included study using the Cochrane Collaboration tool for assessing risk of bias [24] for RCTs and the Ottawa-Newcastle tool for observational studies [25]. Risk of bias assessment will be performed individually per outcome. A third reviewer will be available to resolve any disagreements. For each study, a description for each domain assessed will be included along with comments if necessary and a final judgment. The risk of bias for each study will be categorized as follows: (1) low risk of bias, where bias is not present or if present, unlikely to affect outcomes, (2) high risk of bias, where outcomes are likely to be significantly affected by bias, (3) unclear risk of bias, where there is inadequate reported information to properly assess bias or where it is unclear how much the risk of bias may affect outcomes.

RCTs will be assessed for adequate sequence generation, allocation sequence concealment, blinding, selective outcome reporting, and other bias. Sequence generation will be considered adequate if the study explicitly described an appropriate randomization procedure to generate an unpredictable sequence of allocation, including computerized randomization, use of random number tables, and coin tossing. Concealment of allocation will be considered adequate if specific methods to protect allocation were documented and implemented. Performance bias will be considered low if a study reported participant, caregiver, and/or researcher blinding. Blinding of outcome assessment will be considered adequate if outcome assessors and adjudicators were blinded. Within-study selective reporting of outcomes will be examined by reviewing the a priori study protocol if available. If the study protocol is not available, we will compare the outcomes listed in the "Methods/design" section with those reported in the manuscript.

Observational studies will be assessed for the following: representativeness of the exposed cohort, selection of the non-exposed cohort, ascertainment of exposure, demonstration that the outcome of interest was not present at the start of the study, the comparability of the cohorts on the basis of the design or analysis, outcome assessment methods, and the adequacy of follow-up.

\section{Measures of treatment effect}

When pooling of outcome data is appropriate, RevMan 5.2 software will be used to conduct meta-analyses. RCTs and observational studies will be pooled and analyzed separately. We will use the method of DerSimonian and Liard in random effects model to pool effect sizes for each outcome; study weights will be measured using the inverse variance method. We will present the results as relative risk (RR) with $95 \%$ confidence interval (CI) for dichotomous outcomes and as mean difference (MD) or standardized mean difference (SMD) for continuous outcomes with $95 \%$ CI. We plan to perform random effects analysis for all outcomes of interest. If significant unexplained heterogeneity exists, or if there is an insufficient number of RCTs for meta-analyses, data will be described qualitatively. The number needed to treat (NNT) with $95 \%$ CI will be derived from pooled risk ratios and its $95 \%$ CI utilizing assumed control risk (ACR) for each outcome similar to the approach recommended by the Cochrane collaboration: NNT $=1 /[\mathrm{ACR} \times(1-\mathrm{RR})]$ [26].

\section{Dealing with missing data}

Where possible, if missing data is encountered, we will attempt to contact the individual study authors for additional information. If this is not possible, we will analyze the available data and report any potential impact of missing data on the results in the "Discussion" section.

\section{Assessment of heterogeneity}

We will assess for heterogeneity between studies using the chi-squared test for homogeneity, where $p<0.01$ indicates substantial heterogeneity, and the $I^{2}$ statistic. We consider $I^{2}>50 \%$ to be significant heterogeneity, which will be further investigated with subgroup analyses to assess clinical and methodological sources of heterogeneity in intervention effect. If there is significant statistical or clinical heterogeneity not explained by subgroup or sensitivity analyses, we will not perform a meta-analysis, and instead, we will describe the data qualitatively.

\section{Assessment of reporting biases}

We will look for potential publication bias using a funnel plot if greater than ten trials are included. For continuous outcomes, the Egger test [24] will be used to detect funnel plot asymmetry. For dichotomous outcomes, the arcsine test will be used. All analyses will be performed using RevMan or R.

\section{Subgroup analysis and investigation of heterogeneity}

Potential and expected clinical sources of heterogeneity include different patient demographics, dosing strategies and delivery of clonidine, non-clonidine sedative regimens, and different methods of capture for certain outcomes such as delirium or level of sedation.

To explore significant heterogeneity, when possible, we will conduct the following subgroup analyses: (1) children (under 18 years of age), hypothesizing that the adult population may benefit more from clonidine, given an increased prevalence of opioid or alcohol withdrawal. (2) Clonidine delivered via enteral vs. parenteral route, hypothesizing that there may be issues with enteral delivery of clonidine in the critically ill due to access (for example, requiring a nasogastric tube), absorption, or tolerance concerns leading to greater benefit with 
parenteral delivery. (3) Clonidine delivered once or twice per day vs. more frequent dosing, hypothesizing that the more frequent dosing regimen would provide a more predictable and steady concentration of the drug. (4) Early administration (within the first $48 \mathrm{~h}$ following intubation) vs. later administration (after the first $48 \mathrm{~h}$ following intubation), hypothesizing that earlier use will facilitate weaning or prevent features of withdrawal. (5) Patients who have ongoing opioid or alcohol use leading to a high risk for withdrawal vs. patients without a history of opioid or alcohol use, hypothesizing that withdrawal patients may benefit more from clonidine. (6) Unclear or high risk of bias studies vs. low risk of bias studies, hypothesizing that the studies at high risk of bias will show a larger effect size.

We acknowledge that subgroup analysis may not be possible depending on the number of trials included in the final analysis.

\section{Sensitivity analysis}

A priori sensitivity analysis will be performed excluding studies only reported as abstracts and lacking formal publication. Post hoc sensitivity analysis will be performed where appropriate.

\section{Assessing the quality of evidence}

The Grading of Recommendations Assessment, Development and Evaluation (GRADE) approach will be used to assess the quality of evidence for each outcome [27]. The GRADE system classifies the quality of the aggregate body of evidence as high, moderate, low, or very low. The evidence will be evaluated using the following criteria: (1) study design and rigor of its execution (i.e., individual study risk of bias), (2) the extent to which the evidence could be applied to patients of interest (i.e., directness), (3) the consistency of results, (4) the analysis of the results (i.e., precision), and (5) whether there was a likelihood of publication bias. The following three factors lead to potential upgrading of the quality of evidence if present: (1) a strong or very strong association between an intervention and the observation of interest, (2) a highly statistically significant relationship between dose and effect, and (3) a plausible confounding variable that could explain a reduced effect or could explain an effect if one was not anticipated.

A final overall quality of evidence will be summarized for the intervention taking into consideration both desirable and undesirable outcomes. An evidence profile will be included in the results showing the GRADE assessments and pooled analysis per outcome.

\section{Discussion}

The ideal sedative agent allows for safe and effective sedation practices, thereby potentially reducing the time required to wean from invasive mechanical ventilation and minimizing the use of other sedative-analgesic agents. It should also be associated with minimal adverse effects. Although no perfect agent exists, clonidine may be beneficial compared to available alternatives. This systematic review will summarize the evidence on the efficacy and safety of using clonidine as a sedative agent in critically ill patients. The quality of evidence will be assessed using the GRADE approach to characterize the confidence in the estimate of effect.

\section{Additional files}

Additional file 1: An outline of our search strategy. (XLSX $30 \mathrm{~kb}$ ) Additional file 2: Observational studies data abstraction form. (DOCX $75 \mathrm{~kb}$ )

Additional file 3: RCT data abstraction form. (XLSX $26 \mathrm{~kb})$

\section{Abbreviations}

ACPJC: American College of Physicians Journal Club; ACR: assumed control risk; ADHD: attention deficit hyperactivity disorder; ATS: American Thoracic Society; CAM-ICU: Confusion Assessment Method for the Intensive Care Unit; Cl: confidence interval; CINAHL: Cumulative Index to Nursing and Allied Health Literature; ESICM: European Society of Intensive Care Medicine; GRADE: Grading of recommendations assessment development and evaluation; ICU: intensive care unit; IMV: invasive mechanical ventilation; ISICEM: International Society of Intensive Care and Emergency Medicine; MD: mean difference; NICU: neonatal intensive care unit; NIV: non-invasive ventilation; NNT: number needed to treat; RCT: randomized controlled trial; RR: relative risk; SCCM: Society of Critical Care Medicine; SMD: standardized mean difference; WHO ICTRP: World Health Organization International Clinical Trials Registry Platform.

\section{Competing interests}

The authors declare that they have no competing interests.

\section{Authors' contributions}

BR conceived the idea. JW, EB, and BR designed the search strategy and data abstraction forms. JW, EB, BR, LB, MD, TK, DP, HW, FD, and WA participated in the design of the protocol and contributed to drafting the manuscript. All authors have read and approved the final manuscript.

\section{Acknowledgements}

We would like to express our gratitude to Jean Maragno and Lois Cottrel for their guidance in designing and carrying out our search strategy.

\section{Funding}

There is no dedicated funding for this project.

\section{Author details}

${ }^{1}$ Department of Medicine, Faculty of Health Sciences, McMaster University, Hamilton, Ontario, Canada. ${ }^{2}$ Department of Clinical Epidemiology and Biostatistics, McMaster University, Hamilton, Ontario, Canada. ${ }^{3}$ Department of Pharmacy, Mount Sinai Hospital, Toronto, Ontario, Canada. ${ }^{4}$ Leslie Dan Faculty of Pharmacy, University of Toronto, Toronto, Ontario, Canada.

${ }^{5}$ Department of Pediatrics, McMaster University, Hamilton, Ontario, Canada. ${ }^{6}$ Hamilton Health Sciences, Hamilton, Ontario, Canada. ${ }^{7}$ St. Joseph's

Healthcare Hamilton, Hamilton, Ontario, Canada. ${ }^{8}$ Department of Critical Care Medicine, Sunnybrook Health Sciences Centre, Toronto, Ontario, Canada.

${ }^{9}$ Department of Anesthesia, University of Toronto, Toronto, Ontario, Canada. ${ }^{10}$ Interdepartmental Division of Critical Care Medicine, University of Toronto, Toronto, Ontario, Canada. 
Received: 8 July 2015 Accepted: 19 October 2015

Published online: 06 November 2015

\section{References}

1. Mazzeo AJ. Sedation for the mechanically ventilated patient. Crit Care Clin. 1995;11(4):937.

2. Lewis KS, Whipple JK, Michael KA, Quebbeman EJ. Effect of analgesic treatment on the physiological consequences of acute pain. Am J Health Syst Pharm. 1994;51(12):1539-54.

3. Hansen-Flaschen J. Improving patient tolerance of mechanical ventilation. Challenges ahead. Crit Care Clin. 1994;10(4):659-71.

4. Kress JP, Pohlman AS, O'Connor MF, Hall JB. Daily interruption of sedative infusions in critically ill patients undergoing mechanical ventilation. N Engl J Med. 2000;342(20):1471-7.

5. Barr J, Fraser GL, Puntillo K, Ely EW, Gélinas C, Dasta JF, et al. Clinical practice guidelines for the management of pain, agitation, and delirium in adult patients in the intensive care unit. Crit Care Med. 2013;41(1):263-306.

6. Wunsch $H$, Kahn JM, Kramer AA, Rubenfeld GD. Use of intravenous infusion sedation among mechanically ventilated patients in the United States*. Crit Care Med. 2009;37(12):3031-9.

7. Gerlach AT, Murphy CV, Dasta JF. An updated focused review of dexmedetomidine in adults. Ann Pharmacother. 2009;43(12):2064-74. http://dx.doi.org/10.1345/aph.1M310.

8. Kress JP, Hall JB. Sedation in the mechanically ventilated patient. Crit Care Med. 2006;34(10):2541-6.

9. Fulton B, Sorkin EM. Propofol. An overview of its pharmacology and a review of its clinical efficacy in intensive care sedation. Drugs. 1995;50(4):636-57.

10. Kam P, Cardone D. Propofol infusion syndrome. Anaesthesia. 2007;62(7):690-701.

11. Kollef MH, Levy NT, Ahrens TS, Schaiff R, Prentice D, Sherman G. The use of continuous iv sedation is associated with prolongation of mechanical ventilation. CHEST Journal. 1998;114(2):541-8.

12. Ostermann ME, Keenan SP, Seiferling RA, Sibbald WJ. Sedation in the intensive care unit: a systematic review. JAMA. 2000;283(11):1451-9.

13. Jakob SM, Ruokonen E, Grounds RM, Sarapohja T, Garratt C, Pocock SJ, et al. Dexmedetomidine vs midazolam or propofol for sedation during prolonged mechanical ventilation: two randomized controlled trials. JAMA. 2012;307(11):1151-60.

14. Riker RR, Shehabi Y, Bokesch PM, Ceraso D, Wisemandle W, Koura F, et al. Dexmedetomidine vs midazolam for sedation of critically ill patients: a randomized trial. JAMA. 2009;301(5):489-99.

15. Venn R, Bradshaw C, Spencer R, Brealey D, Caudwell E, Naughton C, et al. Preliminary UK experience of dexmedetomidine, a novel agent for postoperative sedation in the intensive care unit. Anaesthesia. 1999;54(12):1136-42.

16. Hazell $\mathrm{PL}$, Stuart JE. A randomized controlled trial of clonidine added to psychostimulant medication for hyperactive and aggressive children. Journal of the American Academy of Child \& Adolescent Psychiatry. 2003;42(8):886-94.

17. Gold M, Redmond DE, Kleber H. Clonidine blocks acute opiate-withdrawal symptoms. Lancet. 1978;312(8090):599-602.

18. Baumgartner G, Rowen R. Transdermal clonidine versus chlordiazepoxide in alcohol withdrawal: a randomized, controlled clinical trial. South Med J. 1991;84(3):312-21.

19. Wallet F, Clement HJ, Bouret C, Lopez F, Broisin F, Pignal C, et al. Effects of a continuous low-dose clonidine epidural regimen on pain, satisfaction and adverse events during labour: a randomized, double-blind, placebocontrolled trial. European Journal of Anaesthesiology (EJA). 2010;27(5):441-7.

20. Rauck RL, Eisenach JC, Jackson K, Young L, Southern J. Epidural clonidine treatment for refractory reflex sympathetic dystrophy. Anesthesiology. 1993;79(6):1163-9. discussion 27A.

21. Duffett M, Koop A, Menon K, Meade MO, Cook DJ. Clonidine for the sedation of critically ill children: a systematic review. Journal of Pediatric Intensive Care. 2012;1(1):5-15.

22. Jamadarkhana S, Gopal S. Clonidine in adults as a sedative agent in the intensive care unit. J Anaesthesiol Clin Pharmacol. 2010;26(4):439.

23. Chen K, Lu Z, Xin YC, Cai Y, Chen Y, Pan SM. Alpha-2 agonists for long-term sedation during mechanical ventilation in critically ill patients. The Cochrane database of systematic reviews. 2015;1:CD010269. doi:10.1002/14651858.CD010269.pub2.
24. Higgins JP, Altman DG, Gøtzsche PC, Jüni P, Moher D, Oxman AD, et al. The Cochrane Collaboration's tool for assessing risk of bias in randomised trials. BMJ. 2011;343:d5928.

25. Sanderson S, Tatt ID, Higgins JP. Tools for assessing quality and susceptibility to bias in observational studies in epidemiology: a systematic review and annotated bibliography. Int J Epidemiol. 2007;36(3):666-76.

26. Higgins JPT, Green S, eds. Cochrane Handbook for Systematic Reviews of Interventions Version 5.1.0 [updated March 2011]. The Cochrane Collaboration, 2011. Available from www.cochrane-handbook.org.

27. Guyatt GH, Oxman AD, Vist GE, Kunz R, Falck-Ytter Y, Alonso-Coello P, et al. GRADE: an emerging consensus on rating quality of evidence and strength of recommendations. BMJ. 2008;336(7650):924-6. doi:10.1136/bmj.39489.470347.AD.

\section{Submit your next manuscript to BioMed Central and take full advantage of:}

- Convenient online submission

- Thorough peer review

- No space constraints or color figure charges

- Immediate publication on acceptance

- Inclusion in PubMed, CAS, Scopus and Google Scholar

- Research which is freely available for redistribution 